MedienPädagogik

medienpaed.com

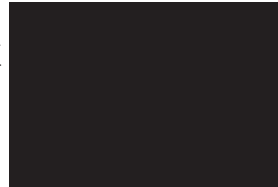

Rudolf Kammerl

19.3.2006

\section{Funktionalität und Dysfunktionalität des Chattens für Beziehungen von 14- bis 16-jährigen Jugendlichen}

Das Motiv, andere Jugendliche kennen zu lernen, ist für Jugendliche der am häufigsten genannte Grund für die Chat-Kommunikation. Ein Treffen mit anderen Chatpartnern wird aber nur für wenige Chatter Realität. Auf der Grundlage einer eigenen qualitativen Studie und unter Berücksichtigung weiterer empirischer Ergebnisse versucht der Beitrag einen Erklärungsansatz für dieses Phänomen zu geben. Die zum Teil in einem Spannungsverhältnis stehenden Erwartungen an die Chatkommunikation führen in Kombination mit strukturellen Besonderheiten der besuchten Webchats dazu, dass es unwahrscheinlich wird, dass für Jugendliche aus Kontakten im Chat längerfristige Beziehungen entstehen.

\section{Erwartungen an einen Chatbesuch und erhaltene Gratifikationen}

Die Frage, ob die erhaltenen Gratifikationen der Mediennutzung den erwarteten Gratifikationen entsprechen, ist nicht nur eine zentrale Fragestellung im Uses-and-Gratification-Approach, sondern betrifft auch die dezidiert medienpädagogische Frage, wie medienkompetent der Nutzer handelt. Die Fähigkeit, eigene Medienhandlungen im Hinblick auf eigene Erwartungen an die Mediennutzung und ihre tatsächlichen Folgen zu reflektieren, zu bewerten und ggf. zu korrigieren, darf - zumindest wenn man die Begriffsbestimmung nach Dieter Baacke zugrunde legt - als elementarer Bestandteil von Medienkompetenz bzw. reflexiver Medienkritik verstanden werden (vgl. z. B. Baacke 1996)
In diesem Beitrag soll die Frage, wie sich die Internetnutzung von Jugendlichen zu deren Erwartungen verhält, in den Mittelpunkt der Ausführungen gestellt werden. Dies wird am Beispiel des Chattens untersucht. Es soll herausgestellt werden, wie Beziehungen und Gemeinschaft in den Zielsetzungen des jugendlichen Chatters enthalten sind und welche Folgen das Chatten nach Aussagen der Jugendlichen für Beziehungen hat. Darüber soll ein Erklärungsversuch entwickelt werden, der auf der Basis der Aussagen von Jugendlichen über ihre Chatroutinen und der Analyse der von ihnen besuchten Chats zeigt, wie das Zusammenspiel von Merkmalen des Kommunikationskanals und subjektive Nutzerroutinen erwartungskonforme Folgen der Chatnutzung unwahrscheinlicher macht.

Jugendliche Chatter wollen andere Jugendliche kennen lernen.

Unter den Jugendlichen, von denen nach den Daten der JIM-Studie 2003 bereits $84 \%$ zu den Internetnutzern zählten, ist das Chatten sehr beliebt. Es nimmt in der insgesamt eher unterhaltungsorientierten Internetnutzung eine besondere Stellung ein, da der Besuch von Chaträumen bei Mädchen und Jungen sehr beliebt ist.

Im Chat kann ich neue Bekannte finden (JIM 2003)

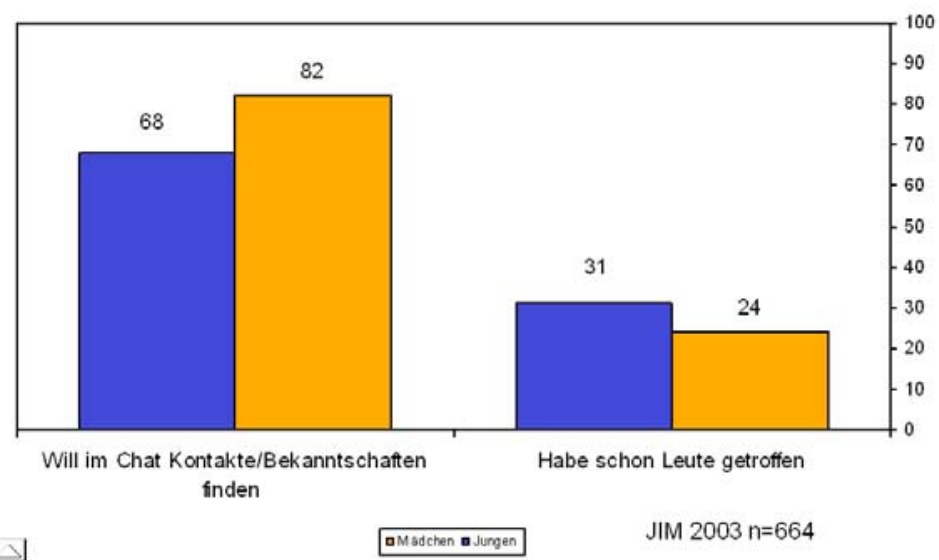

Iab. I: Das Motiv, andere Jugendliche kennen zu lernen und tatsächliche Treffen 
Das Motiv, andere Jugendliche kennen zu lernen, ist der am häufigsten genannte Grund für die Chat-Kommunikation. In der Motivstudie von Fix 2001, in der repräsentativen Befragung im Rahmen der JIM 2003 (vgl. MpFS 2004) und auch in meiner eigenen Studie zeigte sich, dass dies das Hauptmotiv für das Chatten zu sein scheint. Andere Gründe, die relativ häufig genannt werden, sind Unterhaltung, Spass und Zeitvertreib. Der Informationsaustausch spielt eine untergeordnete Rolle.

Wie an den Zahlen der JIM-Studie zu sehen ist, wird ein Treffen mit Chatpartnern - allein oder im Rahmen von Chattertreffen - nur für die Minderheit Wirklichkeit (vgl. MpFS 2004). Der Wunsch nach neuen Kontakten, der das zentrale Motiv für den Chatbesuch ist, wird offensichtlich nur für wenige Wirklichkeit. Das wirft die Notwendigkeit auf, die Funktionalität bzw. die Dysfunktionalität des Chattens für Beziehungen von 14- bis 16-jährigen Jugendlichen differenziert zu betrachten.

\section{Darstellung der Stichprobe und der Methodik der qualitativen Studie}

Von 2001 bis 2003 wurden von uns in einer explorativen, qualitativen Studie 32 Jugendliche im Alter von 14-16 begleitet, die 2001 innerhalb ihrer Altersgruppe den «Vielnutzern» zugerechnet werden konnten. «Vielnutzer» ist hierbei als relativer Begriff zu verstehen. Konnten 2001 noch Jugendliche, die täglich oder mehrmals pro Woche das Internet nutzten und im Durchschnitt zehn Stunden pro Woche oder mehr online waren, im Vergleich zu Internetanwendern in dieser Altersgruppe als «Vielnutzer» bezeichnet werden, so waren diese Nutzungszeiten bereits 2003 nicht mehr geeignet, da sich die durchschnittliche Internetnutzung in dieser Altersgruppe deutliche erhöht hatte. Um bei der Stichprobe eine hohe subjektive Relevanz der Internetnutzung sicherzustellen, wurden die Jugendlichen gebeten, ihre Freizeitaktivitäten geordnet nach Wichtigkeit in eine Rangreihe zu bringen. Nur Jugendliche, auf deren Rangliste das Internet einen der vorderen drei Plätze innehatte, wurden gewählt. Weitere Kriterien sind der regelmässige Besuch von Chats oder MUDs und eine eigene Homepage. Die Jugendlichen waren in Schulen und Internetcafés in Niederbayern zur Teilnahme eingeladen worden.

Mit jedem Teilnehmer der Studie wurden mindestens zwei halbstrukturierte Interviews durchgeführt, die dann inhaltsanalytisch ausgewertet wurden. In den von den Jugendlichen regelmässig besuchten Chats wurden systematisch Beobachtungen durchgeführt und Daten über die Rahmenbedingungen der Chatkommunikation erhoben. Dazu gehörten insbesondere Regel- werke und Protokolle zu Regelverstössen und Operatorentätigkeiten. 19 Probanden nutzen 2001 regelmässig den Chatraum «Passau» des Radiosenders Antenne Bayern. Dadurch ergab sich eine Fokussierung auf diesen Chat. Mittels Triangulation wurden die verschiedenen Daten in Beziehung gesetzt.

$\mathrm{Da}$ in der Entwicklungspsychologie insbesondere die Aufnahme und Gestaltung sozialer Beziehungen zu Peers für das Jugendalter als Entwicklungsaufgabe angesehen wird, bildete in den Interviews die Frage, welche Funktionen die internetbasierte Kommunikation für Beziehungen hat, einen Schwerpunkt.

Die Jugendlichen wurden hierzu vor allem zu folgenden Bereichen befragt: (Entwicklung der) Zugehörigkeit zu einer Online-Community, Veränderungen der Beziehungen zu Freunden und Familie, sowie Entstehung und Entwicklung von Beziehungen der Jugendlichen zu anderen Chat-Besuchern (auch offline). Der zuletzt genannte Aspekt soll in den Vordergrund gestellt werden:

\section{Von Jugendlichen benannte Folgen der Chatkommunikation}

Blickt man auf die von den Jugendlichen genannten Folgen der Chat-Kommunikation, so zeigt sich, dass sich aus Chat-Kontakten durchaus subjektiv relevante, längerfristige Sozialbeziehungen entwickeln können. Empathie und Bindung im Einzel- oder Gruppenkontakt können zu prosozialen Orientierungen führen, aus denen auch soziale Beziehungen ausserhalb des Netzes entstehen können. Die Selbstbeobachtungen der Jugendlichen sind aber keineswegs gleichzusetzen mit Wirkungen des Chattens, aber sie verdeutlichen, mit welchen Prozessen das Zusammenspiel von (medialer) Umwelt und Person intern organisiert und nach aussen hin dargestellt wird. In der Betrachtung der verschiedenen Nennungen der Jugendlichen wird deutlich, dass von den Jugendlichen sowohl langfristig wie auch kurzfristig beabsichtigte und unbeabsichtigte Konsequenzen benannt wurden. Die Jugendlichen schreiben der Chat-Kommunikation sowohl bereichsspezifische (z. B. Anerkennung in der Chat-Community) wie auch bereichsfernere Entwicklungen (z. B. Belastung der Beziehung zu den Eltern) zu. Sowohl positiv bewertete Veränderungen (Beherrschung des Zehnfingersystems beim Tippen) wie auch negative Entwicklungen (Abkapselung vom sozialen Umfeld) wurden benannt (vgl. Tab. 1). 


\begin{tabular}{|l|l|l|}
\hline & Kurzfristig & Langfristig \\
\hline Beabsichtigt & $\begin{array}{l}\text { Unterhaltung, Spass, Auf- } \\
\text { merksamkeit, positive Rück- } \\
\text { meldungen, neue Kontakte, } \\
\text { kostengünstige Kommunika- } \\
\text { tion unter Freunden }\end{array}$ & $\begin{array}{l}\text { Kennenlernen von Peers, neue } \\
\text { Freunde, Partner, Zugehörigkeit zu } \\
\text { einer Gemeinschaft, Anerkennung, } \\
\text { Einfluss }\end{array}$ \\
\hline Unbeabsichtigt & $\begin{array}{l}\text { Erlebnis der Selbst- } \\
\text { wirksamkeit, Langeweile, } \\
\text { Ärger, Frust, Missachtung, } \\
\text { Ignorierung, negative } \\
\text { Erfahrungen, Enthemmung, } \\
\text { Übertretung des selbst } \\
\text { gesteckten zeitlichen } \\
\text { Rahmens }\end{array}$ & $\begin{array}{l}\text { Belastung sozialer Beziehungen, } \\
\text { Zeitmangel (z. T. - - Gefährdung des } \\
\text { Schulerfolgs), Kontrollverlust, } \\
\text { Änderung des Selbstkonzepts (Ich als } \\
\text { Chatter) und Selbstbewertung, } \\
\text { Kennenlernen sozialer und } \\
\text { technischer Bedingungen des Chats, } \\
\text { Erwerb des Chatslangs, Aufbau von } \\
\text { Chat-Routinen, gesteigerte } \\
\text { Fertigkeiten im Tippen. }\end{array}$ \\
& & \\
\hline
\end{tabular}

Tab. 2: Benannte kurzfristige und langfristige Folgen von Chat-Kommunikation

Die Bejahung der Frage, ob der Chat einen Möglichkeitsraum darstellt, in dem soziale Beziehungen aufgebaut werden könnten, ist allerdings nicht gleichzusetzen mit der Antwort auf die Frage, ob Jugendliche untereinander ausgehend von der Chat-Kommunikation tatsächlich stabile Beziehungen aufbauen.

\begin{tabular}{|l|l|l|}
\hline $\begin{array}{l}\text { Subjektiv relevante, länger- } \\
\text { fristige (länger als drei Monate) } \\
\text { Kontakte zu Internet- } \\
\text { bekanntschaften }\end{array}$ & 2001 & $\begin{array}{l}\text { 2003 } \\
\text { (In Klammern sind die } \\
\text { Bekanntschaften angegeben, } \\
\text { die auch schon 2001 } \\
\text { bestanden) }\end{array}$ \\
\hline $\begin{array}{l}\text { Hohe Relevanz (z. B. Be- } \\
\text { ziehung zu Partner, enge } \\
\text { Freundschaft o. ä.) }\end{array}$ & 4 & $2(0)$ \\
\hline $\begin{array}{l}\text { Mittlere (z. B. Freund, Spiele- } \\
\text { partner o. ä.) }\end{array}$ & 5 & $3(1)$ \\
\hline $\begin{array}{l}\text { Keine, bzw. geringe Relevanz } \\
\text { (z. B. sporadisch, aber } \\
\text { kontinuierlicher Kontakt) }\end{array}$ & 7 & $5(2)$ \\
\hline $\mathrm{N}$ & 8 (aus 32) & 7 (aus 28) \\
\hline
\end{tabular}

Tab. 3: Längerfristige Beziehungen mit Internetbekanntschaften (Mehrfachnennungen möglich)
Die Jugendlichen wurden befragt, inwiefern sich aus den Chat-Bekanntschaften oder aus anderen Internetbekanntschaften längerfristige, stabile Beziehungen (online und/oder offline) entwickelt haben.

Insgesamt war dies nur bei einer Minderheit der Fall. Zu berücksichtigen ist, dass bei der Gruppe der Jugendlichen, die angaben, schon seit längerem feste Kontakt(e) zu bestimmten Personen zu haben, die sie im Chat kennen gelernt hatten, sich sowohl die Zusammensetzung wie auch die genannten Bezugspartner verändert hatten. D. h. Beziehungen, die sich 2001 etabliert hatten, waren 2003 nicht mehr aktuell. Neue Freundschaften hatten sich entwickelt. Bereits entstandene Beziehungen verliefen wieder im Sande.

Bezogen auf einzelne Fälle zeigt sich, dass etliche Jugendliche, die chatten, «um neue Leute kennen zu lernen» o. ä., nach eigenen Angaben mehrere Monate bzw. Jahre im Schnitt bis zu 20 Stunden pro Woche den Chat besuchen, ohne dass sie jemand aus dem Chat getroffen hätten und/oder sich langfristig ein fester Kontakt zu einem Chat-Partner entwickelt hätte. Zwar chatten auch diese Jugendlichen mit einer Vielzahl von Kommunikationspartnern, aber aus diesen virtuellen Gesprächen entwickeln sich nur selten Kommunikationssequenzen, die über mehrere Sitzungen hinausgingen und/oder über den Chat hinaus weitergeführt wurden. Dies war gerade deshalb bemerkenswert, da die absolute Mehrheit der Stichprobe (30 von 32 VPN) durchaus bestätigte, über dem Kontakt im Chat an einem Kennenlernen Face-to-Face interessiert zu sein. Für eine grosse Gruppe war dies auch der Grund Chaträume zu wählen, die einen lokalen Bezug zu ihrem Wohnort hatten'.

Wie sind diese Schwierigkeiten, mit Hilfe des Chattens Beziehungen zu knüpfen, zu erklären? Ist der Chatraum eher ein Spielplatz für Identitätsexperimente als ein Ort an dem Kontakte gesucht werden? Anhand der Chatroutinen, welche die befragten Jugendlichen berichteten und auf der Grundlage eigener Erhebungen im Chat lassen sich einige Aspekte benennen, welche - selbst dann, wenn zwei Jugendliche mit derselben Motivlage aufeinander treffen - die Wahrscheinlichkeit gering halten, dass aus einer Begegnung im Chat ein weitergehender Kontakt entsteht.

\footnotetext{
${ }^{1}$ Alle der befragten Jugendliche, die im Internet Bekanntschaften geschlossen hatten, zeigten den Wunsch nach realen Treffen. Deutliche Unterschiede zeigten sich darin, wie lange ein Kontakt erst im virtuellen Raum gepflegt werden sollte, bevor ein Faceto-Face-Treffen angestrebt wurde und wie viele der Internetbekannten auch hierfür ausgewählt wurden. 
Chatten - nur ein Spiel mit der Identität?

Gerade der Möglichkeit, im Internet ganz oder teilweise eine andere Rolle zu spielen, wurde in der Fachliteratur hohe Aufmerksamkeit gewidmet. Zwar sitzt der Internetnutzer «nach wie vor mit seiner ganzen Körperlichkeit vor dem Bildschirm, betätigt motorisch Tastatur und Maus, aber in den sich elektronisch öffnenden virtuellen Welten kann er für sich und vor allem für andere eine andere Identität annehmen. Er kann mit Identitäten spielen oder sich gar als nicht-menschliches Wesen ausgeben und fühlen» (Kübler 2001, S. 17). Spätestens seit Sherry Turkles internationalem Erfolg mit der Monografie «Life on the Screen» (1995) wird auch im deutschsprachigen Raum immer wieder die Frage gestellt, «(...) ob solche unermüdlichen Surfer in den virtuellen Welten noch ihre reale physische und soziale Existenz genügend wahrnehmen und wertschätzen oder [sich] zeitweilig in die Cyberspaces verabschiedet haben» (ebd.). Da Turkle die Selbsterfahrung der Internetnutzer in diesen Welten als funktional für die Ausbildung multipler Identitätskonstruktionen auch bei Kindern und Jugendlichen darstellt, war zu erkunden, welche Funktionen die Internetnutzung für die Jugendlichen dieser Stichprobe hat.

Inwiefern nutzen Jugendliche die Möglichkeiten, sich als jemand ganz anderes darzustellen oder in Teilaspekten andere Angaben zu machen? Da die Anonymität des Chat-Raumes und die technischen und sozialen (Regeln) Bedingungen für den Chat der Umsetzung eines entsprechenden Vorhabens nicht im Wege stehen, sondern begünstigen, wäre es für die Jugendlichen einfach, sich im Chat anders darzustellen als es ihrem Selbstbild entspricht. Neben diesen beabsichtigten Veränderungen des Selbstdarstellungsverhaltens war aber ausgehend von entsprechenden Befunden zur computervermittelten Kommunikation auch zu überprüfen, inwiefern die Jugendlichen an sich Veränderungen im Kommunikationsverhalten feststellen, die nicht auch auf eine beabsichtigte (Teil)Maskierung zurückzuführen sind. Dass sich Chatter im Chat anders verhalten als in der Faceto-Face-Kommunikation, kann im Sinne deren Selbstbeobachtung eher als Folge der Chat-Kommunikation interpretiert werden statt als konsequente Maskierung.

Die Interviews mit den Jugendlichen zeigten, dass nach deren Angaben beabsichtigte Veränderungen der Selbstdarstellungen nur in einem geringeren Masse stattfinden. Die befragten Jugendlichen nehmen die Möglichkeiten der Täuschung nur in einem begrenzten Umfang wahr. Nach ihren Angaben geben einige sich nur in Einzelfällen als jemand ganz anderes aus.
Eine Minderheit verändert regelmässig Teilaspekte. In den meisten Fällen geben sich die Jugendlichen so, wie sie sich sonst auch ausserhalb des Internets verhalten.

Andererseits ist zu berücksichtigen, dass die Rolle des Chatbesuchers auch bei den Jugendlichen typische Kommunikationssequenzen induziert, die für den Chat typisch sind. Die standardisierte Abfrage von Geschlecht, Alter und Wohnort wird auch von den befragten Jugendlichen als deren typische Eröffnungssequenz bei neuen Chatkontakten bestätigt. Damit aber zeigen sie zwar bereits ein völlig anderes Verhalten als in vergleichbaren Face-toFace-Situationen. Diese Differenz wird aber von den Jugendlichen selbst aber offensichtlich nicht als relevant betrachtet.

Wenn sich die Jugendlichen im Internet als jemand völlig anderes darstellten, dann um bestimmte Chat-Partner zu necken und selbst Spass dabei zu haben. Es handelte sich bei den berichteten Episoden um vereinzelte Spielereien. Die Jugendlichen spielen die ausgedachte Rolle nur in einer Sitzung und nicht über einen längeren Zeitraum hinweg. Häufig verwendeten die betreffenden Jugendlichen hierbei einen anderen Nickname und/oder einen anderen Chat-Raum, um auszuschliessen, dass sie in ihrem Stamm-Chat bzw. unter ihrem hauptsächlich verwendeten Nick mit negativen Konsequenzen zu rechnen haben.

Die von den Jugendlichen berichteten Beispiele erinnern an Jugendstreiche, die auch aus dem Kontext des «Telefonterrors» bekannt sind.

Michael (15) erzählt: «Vorletzter Schultag, da haben wir den ganzen Tag im Internet sein dürfen und dann sind wir in den Chat gegangen und haben wir Leute verarscht. Erst haben wir uns als Lesben ausgegeben, dann haben wir als Omas geschrieben: <ich bin 80 Jahre alt, habe meine Katze in die Wachmaschine gesteckt und eingeschaltet>.»

Simone (14) berichtet, dass sie schon lustige Sachen erlebt hat. Im Flirt ab 40 haben sie und ihre Freundin sich als Hildegard und Rosemarie (50 Jahre) ausgegeben. Einmal bezeichnete sie sich als «Australier mit Kängurufarm und die haben mir das alles geglaubt.»

Andere Chat-Besucher «für dumm verkaufen» macht einigen Jugendlichen schon mal Spass. Die Erfahrung, bei Chat-Partnern einen gewünschten Eindruck erfolgreich erzeugen zu können, ist als Kontrollerfahrung für die Einschätzung eigener kommunikativer Kompetenz und der des Gegenübers bedeutsam, impliziert aber nicht, sich als multiple Identität (Teilselbst 
Känguruzüchter) wahrzunehmen. Es handelt sich hier um einzelne Erlebnisse, die von den Nutzungsroutinen der jeweiligen Internetnutzer abweichen.

Zwar wird in der Literatur häufig auf das Phänomen des Genderswitch / Genderswapping hingewiesen. Aber im Alltag der Chat-Kommunikation dürfte diese Form nur eine untergeordnete Rolle haben. In der Stichprobe waren keine Jugendlichen, die öfters unter einem anderen Geschlecht am Chat teilnahmen.

Sonja beispielsweise gab im Interview an, einmal als Junge den Chat besucht zu haben, weil sie einfach mal sehen wollte, «wie die Mädels so schreiben». In der Regel macht sie allerdings immer die richtigen Angaben. «Das bringt mir ja nichts, wenn ich da was anderes reinschreib'», meinte sie.

Wesentlich häufiger fanden Veränderungen bei den Altersangaben statt. Insbesondere weibliche Jugendliche führten in den Interviews aus, dass sie regelmässig im Chat ein falsches Alter angeben würden. Analog zum Geschlechtertausch könnte dieses Phänomen als Ageswapping bzw. Ageswitching bezeichnet werden.

Um für ältere (in der Regel männliche) Kommunikationspartner als Gesprächspartner attraktiv zu sein, gaben die Mädchen häufig ein höheres Lebensalter an. Andere Merkmale wurden nicht bewusst verändert. Diese Altersangabe konnte im Text gemacht werden oder war bereits im Nickname enthalten. Simone beispielsweise verwendete vor ihrem 14. Geburtstag in der Regel den Nickname «Piepmatz14», danach aber nicht mehr. Bei dieser Form der Manipulation dürfte es sich um ein jugendspezifisches, nicht um ein internetspezifisches Phänomen handeln. Dass sich Jugendliche gerne etwas älter ausgeben, damit ihnen die notwendige Reife für bestimmte Güter oder Aktivitäten zugeschrieben wird, ist auch ausserhalb des Internets überall dort bekannt, wo formal oder informell geregelt Altersbeschränkungen existieren (Alkoholausschank, Disko, Kino etc.). Das Erlangen von Freiheiten, die durch Altersgrenzen (statt unter Berücksichtigung des individuellen Entwicklungsstands) geregelt werden, ist für die Jugendlichen ein Thema, das ihre Entwicklungsphase begleitet, und deshalb war es auch nicht verwunderlich, dass viele der Jugendlichen angaben, dass sie gerne etwas älter sein würden und diesen Wunsch mit dem Zugewinn an einschlägigen Handlungsspielräumen begründeten («dann darf ich rauchen», «da kann ich mir ein Auto kaufen», «dann darf ich endlich fortgehen» etc.). Auch Jungen gaben an, gerne etwas älter zu sein. Allerdings führte dieser Wunsch bei den befragten Jungen in der Regel nicht dazu, sich im Chat älter darzustellen. Dass die Bereitschaft, das eigene Alter zu modifizieren, um im Rahmen der Chat-Kommunikation für gegengeschlechtliche Kommunikationspartner bei Jungen und Mädchen unterschiedlich ausgeprägt ist, muss im Rahmen der geschlechtsspezifischen Sozialisation als Junge bzw. als Mädchen interpretiert werden. Das Bemühen, den Erwartungen des anderen Geschlechts zu entsprechen, ist im Jugendalter bei Mädchen generell stärker ausgeprägt als bei Jungen (vgl. Fend 1994, S. 124ff).

Mit dem Ageswitching nehmen die jungen Chat-Besucherinnen ihr späteres Alter vorweg, um mit anderen Chat-Partnern in Kontakt zu kommen. Insofern könnte dieses Verhalten als gezieltes Training für ihre zukünftige Rolle als junge Frau interpretiert werden (vgl. «identity workshop» Bruckman 1996). Da aber die betreffenden Interviewpartnerinnen befragt nach dem Hintergrund ihrer Altersänderung dieses Motiv nicht nennen, kann diese Absicht auch nicht unterstellt werden. Möglicherweise handelt es sich statt einem selbst initiierten Trainingsprogramm lediglich um eine Funktion der Chat-Kommunikation, durch welche die Mädchen Erfahrungen sammeln, die später bzw. offline für den Umgang mit dem anderen Geschlecht bedeutsam werden. Die Intention ist es aber lediglich, als Kommunikationspartner akzeptiert $\mathrm{zu}$ werden und als Chat-Partner angenommen zu werden ${ }^{2}$. Dieser feine Unterschied kann für Selbstbewertung des eigenen Verhaltens durchaus bedeutsam sein. Wird ein an sich selbst wahrgenommenes «Defizit» durch eine falsche Angabe kaschiert, um den Erwartungen der männlichen Kommunikationspartner gerecht zu werden, oder ist der Austausch mit diesen ein geeignetes Mittel für das subjektive Kommunikationstrainingsprogramm? Für die Mädchen aus der Stichprobe scheint eher Ersteres zuzutreffen.

Eine erste quantitative Studie mit Jugendlichen, welche diese Interpretation stützt, findet sich bei Schatz (2003, S. 82ff). Das Motiv, im Chat einmal so

2 Die asymmetrische Verteilung der Geschlechter im Internet, die noch fünf Jahre zuvor zu einem Frauenmangel in den Chats führte, war in den Jahren 2001 bis 2003 nicht mehr so deutlich ausgeprägt. Ausgehend von den Schwierigkeiten bei jüngeren Chat-Besucherinnen geeignete Teilnehmer zu finden, wäre es denkbar, dass gerade in der Chat-Kommunikation bei den 11- bis 14-jährigen Chat-Besuchern die Mädchen in der Überzahl sind. Hinweise hierzu liefern JIM 2002, KIM 2002, Schatz 2003 und Hoffmann/ Münch 2003. 
sein zu können, wie man gerne möchte, zeigt sich vor allem bei den Mädchen der 7. und 8. Klassen und zwar insbesondere bei denen, die mit sich selbst unzufrieden sind.

Das zentrale Motiv, einen Chat zu besuchen, ist der Wunsch mit anderen Internetnutzern zu kommunizieren ${ }^{3}$. Dies wird nicht automatisch durch das Einwählen erreicht, sondern erfordert Aktivitäten. Um überhaupt längere Kommunikationssequenzen mit anderen Chat-Besuchern führen zu können, ist ein gewisses Mass an Schnelligkeit, Schlagfertigkeit und Originalität erforderlich, mit der man sich im Hauptfenster oder in die Dialogfenster einbringt. Um einen Kommunikationsabbruch durch das Gegenüber zu vermeiden, ist eine hinreichende Anpassung an dessen Erwartungen erforderlich. Wie im Alltagsgespräch ist auch im Chat die eigene Identität Ausgangs- und Referenzpunkt von Kommunikation. Verstellt sich ein Chatter zu sehr, wird dies nicht nur anstrengend, sondern bringt für ihn auch keinen Nutzen, da die Kommunikationspartner sich auf eine fingierte Person beziehen und nicht auf einen selbst (vgl. Scherer/Wirth 2002, S. 352). Im Fall der Altersangabe kann die Abweichung vom tatsächlichen Geburtsalter von den betreffenden Mädchen häufig recht gut mit ihrer Selbstwahrnehmung harmonieren. So wird betont, dass sie auch ausserhalb des Internets auf andere schon viel älter wirken würden, dass sie schon weiter entwickelt seien etc. Kleinere Übertreibungen und Untertreibungen, das Ausblenden, Hinzuerfinden oder Betonen verschiedener Teilaspekte sind auch in der Alltagskommunikation übliche Formen des Impression Managements, das schon allein aus der Notwendigkeit der Komplexitätsreduktion zu erklären ist.

Kleinere Veränderungen in der Selbstdarstellung, die Scherer/Wirth (2002) bei zwei Drittel aller Chatter feststellen und die in unserer Stichprobe von zehn Jugendlichen eingeräumt wurden, führen nicht dazu, dass sich die Chatter als nicht mehr authentisch erleben. Scherer und Wirth schliessen auf «beziehungs- und kommunikationstaktische Gründe» (a.a.O., S. 354).

Dass sich keiner der Jugendlichen über einen längeren Zeitraum absichtlich ganz anders dargestellt hat als es seinem Selbstbild entspricht, kann aber auch auf Motive zurückgeführt werden, die über die Kommunikation im Chat hinausgehen. Spätestens dann, wenn der Wunsch entsteht, auch ausserhalb der internetbasierten Kommunikation Kontakt zu dem Chat-

\footnotetext{
3 Sowohl das Probehandeln, der Spass wie auch das Kennenlernen lassen sich darunter subsumieren.

Partner aufzunehmen, drohen Diskrepanzen zwischen den Angaben im Chat und offensichtlichen Tatsachen zu Tage zu treten. Tatsächlich stellt sich dieser Übergang für die Beziehung der Internetnutzer zum Teil als sehr kritische Phase dar (vgl. z. B. Bahl 1997, S. 103). In der Antizipation einer Kontaktaufnahme ausserhalb des Chats ist es für die Chat-Besucher, die daran interessiert sind, geboten, sich einerseits so attraktiv darzustellen, dass sie als Kommunikationspartner von Interesse sind, und zum anderen so authentisch darzustellen, dass diese Darstellung einer möglichen Überprüfung auch in Face-to-Face-Situationen standhält. Im Falle der Mädchen, die sich älter ausgeben, könnte dieser Übergang als eine überwindbare Barriere betrachtet werden, da sie glauben, dass sie älter bzw. entwickelter aussehen und auch im Chat älter bzw. reifer wirken, als dies aufgrund der Alterangabe von den Chatpartnern vermutet werden würde.

Die Motive für die Chat-Kommunikation, die von den Jugendlichen genannt wurden, lassen sich in verschiedene Bereiche zusammenfassen. Die Befragten besuchten den Chat, um Spass zu haben (gegen Langeweile), damit sie andere Jugendliche kennen lernen, und um mit ihren Freunden (insbesondere im ICQ) zu kommunizieren.

Nur das Spassmotiv allein würde eine umfassendere Maskierung für die Chat-Besucher nahe legen, die aus dem Necken anderer bzw. dem Rollenspiel Freude ziehen können. Aber auch hier ist der Nutzen der ChatKommunikation spätestens dann beendet, wenn sie vom Gegenüber abgebrochen wird. Wer mit seinem festen Nickname oder seiner ICQ-Adresse via Chat mit Peers kommuniziert, die er bereits ausserhalb des Chats kennt, dem bleibt das Changieren mit Identitätsmerkmalen weitgehend vorenthalten, da er schon identifiziert ist. Das Motiv, durch die internetbasierte Kommunikation neue Kontakte zu knüpfen und über den Chat hinausgehende persönliche Beziehungen aufzubauen, war insbesondere für die Besucher des Chat-Raumes Passau im Antenne Bayern Chat von hoher Bedeutung. Es kann angenommen werden, dass dieses Motiv für die Wahl des Chat-Raumes bedeutsam ist. Der Raumname «Passau» legt die - mit der Historie des Chats konforme - Erwartung nahe, hier auf andere ChatBesucher aus der näheren Umgebung zu treffen. Die Wahl des ChatRaumes stellt deshalb gerade für die Internetnutzer, die an Face-to-FaceKontakten interessiert sind, insofern eine «rational choice» dar, da die Kosten, eine Beziehung mit einem Chat-Partner aus einer weiter entfernten Region aufzunehmen und zu pflegen, wesentlich höher sind. Es ist anzunehmen, dass ausgehend von der subjektiven Erwartung über die 
Zusammensetzung der Besucher (regionaler Bezug, Alter, thematische Interessen etc.) eine Selbstselektion stattfindet und diese begünstigt im Fall eines regionalen Bezugs die Aktivierung des Motivs, über den Chat hinaus Kontakt aufzunehmen.

Auch wenn die Bedingungen der Chat-Kommunikation nur von einem Teil der Chat-Besucher genutzt werden, um sich anders darzustellen als sie zu sein glauben, sind neben den benannten Einzelfällen und den partiellen absichtlichen Modifikationen auch Veränderungen zu berücksichtigen, die Chat-Besucher an sich selbst beobachten, aber nicht von ihnen intendiert waren.

Chat-Kommunikation führt bei einigen Chat-Besuchern zu Verhaltensänderungen, die für die Einschätzung ihrer Identitätsmerkmale durch ihre Chat-Partner bedeutsam sind. Elf der Jugendlichen, die häufig einen Chat besuchten, berichteten davon, dass sie sich in der Kommunikation mit den Chat-Partnern anders verhalten als in Face-to-Face-Situationen. Diese Mädchen und Jungen gaben an, dass sie im Chat «freier», «frecher», «weniger schüchtern», bzw. «offener» auftreten würden. Da «traut man sich mehr». «Man kommt einfach schneller in ein Gespräch hinein» als in einer Face-to-Face-Situation. Ein Jugendlicher gab an, dass er im Chat aggressiver auftrete als ausserhalb des Chats:

Zusammenfassend lässt sich ausgehend von diesen Angaben von einer enthemmenden Funktion sprechen, welche die Chat-Kommunikation für diese Jugendlichen hat. Auch in der Betrachtung der Besucher des ChatRaums Passau des Antenne-Bayern-Chat lässt sich feststellen, dass bei nahezu identischen Rahmenbedingungen das Phänomen der Enthemmung nur von einem Teil der Chat-Besucher berichtet wurde. Die Mehrheit gab aber an, dass sie sich in der Kommunikation auch nicht anders verhalten würden als in anderen Situationen ausserhalb des Internet ${ }^{4}$.

\footnotetext{
${ }^{4}$ Natürlich erfordert die synchrone Kommunikation mit Schrifttext signifikant andere Verhaltensweisen als ein Gespräch unter vier Augen. Die Chat-Sprache weist - trotz Ähnlichkeiten - signifikante andere Merkmale auf als das gesprochene Wort. Wenn also Chatter angeben, sie verhielten sich im Chat nicht anders als in Face-to-FaceSituationen, dann beziehen sie sich auf Gesprächshaltungen, die sie einnehmen.

Zeigen Personen bei vergleichbaren Rahmenbedingungen über einen längeren Zeitraum stabile Verhaltensweisen, ist es nahe liegend, die Verhaltensunterschiede zwischen den Personen auf Persönlichkeitsmerkmale zurückzuführen. Aus der Perspektive des Beobachters wären also internale Bedingungen anzunehmen, die unter den Bedingungen der Chat-Kommunikation zu einer Enthemmung führen. Die betreffenden Jugendlichen selbst erklärten ihr unterschiedliches Verhalten jedoch mit

Spätestens dann, wenn die Chat-Kontakte dieser Jugendlichen in persönliche Beziehungen überführt werden sollen, die über den Chat hinausgehen, könnten diese Diskrepanzen bemerkt und zum Kommunikationsabbruch führen.

\section{Behindern die Strukturen von Chatkommunikation \\ die Entwicklung von Beziehungen?}

Wie in den Ausführungen der Befragten deutlich wurde, gibt es nach dem Betreten des Chat-Raumes unterschiedliche Vorgehensweisen der Besucher. Die Möglichkeit, sich im Hauptfenster zu beteiligen, wird nur von einem Teil der Chat-Besucher genutzt. Nur die Minderheit bleibt hier über die ganze Sitzung engagiert. Viele nutzen das Hauptfenster nur, um Gesprächspartner für private Dialoge zu rekrutieren (Wer hat Lust zum Chatten? u. ä.). Andere versuchen gleich nach dem Eintreten über Dialogfenster zu anderen Chat-Besuchern Kommunikationssequenzen zu initiieren. Die Suche nach interessierten Chat-Partnern im Chat stellt keine einfache Aufgabe dar (vgl. Orthmann 2001, S. 297) , die zwar Wartezeiten verursachen kann, aber für die befragten Vielnutzer in der Regel ohne Mühe gemeistert wird. Die ersten Schritte in der privaten Kommunikation zwischen zwei Chat-Partnern sind sehr standardisiert. Nach der Begrüssung werden in der Regel gegenseitig zunächst (bei unklarem Nick: Geschlecht) Alter, Wohnort, Aussehen und Interessen/Hobbys abgefragt. ${ }^{6}$ Jede der Angaben kann zum Abbruch des Dialogs führen. Die Jugendlichen haben für sich einen Erwartungshorizont definiert, mit dem sie anhand dieser Selbstauskünfte des Gegenübers entscheiden, ob sie an einem längeren

den verschiedenen externen Bedingungen von Chat und Face-to-Face-Kommunikation (im Chat, da sieht man sich nicht, da ist nicht mit Konsequenzen zu rechnen usw.). Die Befragten nahmen also eine externale Attribuierung vor. Damit vermieden sie es in den Interviews interne Variablen als mögliche Ursachen darzustellen. Mit solchen geäusserten Kontrollüberzeugungen hinsichtlich der Chat-Kommunikation findet sich kein Hinweis auf eine Unterscheidung in verschiedene Teilselbste. Wenn Individuen für sich unterschiedliches Verhalten mit verschiedenen äusseren Bedingungen erklären können, stützen sie die Integrität und Kontinuität ihres Selbstkonzepts. Von aussen betrachtet hält diese Deutung allerdings nicht stand.

5 Deswegen wird die Aufmerksamkeit der Chat-Partner auch als «Währung des Chats» bezeichnet (Wolf/ Bilandzic 2003).

${ }^{6}$ Anders als bei den Kindern und Jugendlichen aus der Stichprobe von Orthmann hatte der Name in dieser Stichprobe nach den Angaben der befragten Jugendlichen nur nachrangige Bedeutung (vgl. Orthmann 2001, S. 300). 
Gespräch interessiert sind. Eine Vielzahl von Dialogen geht über diese Anfangssequenzen (Age/Sex/Location-Check) nicht hinaus. Damit ist ein hoher Anteil in der Chat-Kommunikation der Jugendlichen hoch standardisiert.

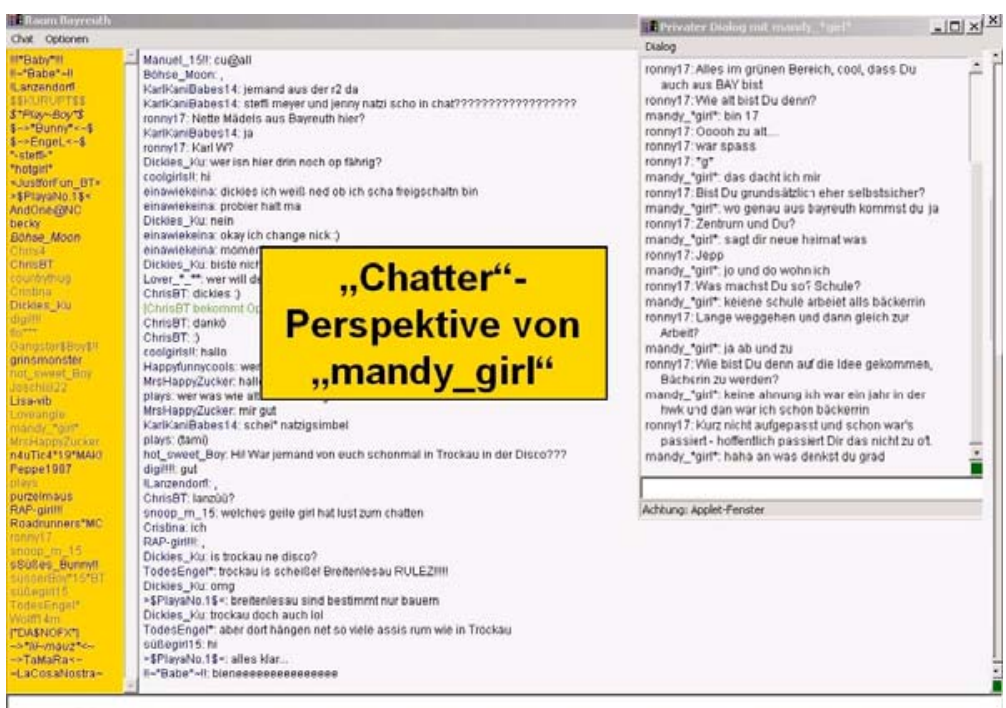

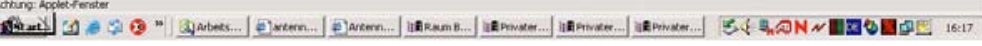

Abb. 1: Chatbesuch von mandy_girl (nachgestellt) mit einem geöffneten Dialog (mit ronny17)

Die Möglichkeit, mehrere Dialoge gleichzeitig zu führen, wird unterschiedlich genutzt. In der Stichprobe fanden sich sowohl Jugendliche, die für gewöhnlich nur einen Dialog führen, wie auch Jugendliche, die angaben, mehrere Dialoge (bis zu acht parallel geöffnete Dialogfenster) gleichzeitig zu bedienen. Da die Dialoge zwischen den Chat-Partnern jederzeit und aus Gründen, die für das Gegenüber nicht erkennbar sind, abgebrochen werden können, erhöht eine höhere Anzahl von Dialogen, die ein Chat-Besucher geöffnet hat, zum einen die Wahrscheinlichkeit einen Chat-Besucher zu treffen, der für sympathisch erachtet wird, und zum anderen kann die Geschwindigkeit, mit der die Tastatur bedient wird, so individuell reguliert werden. Mit einem offenen Dialog ist die Ge- schwindigkeit der Kommunikation von der Reaktionsschnelligkeit bzw. freudigkeit eines Chat-Partners abhängig. Eine höhere Anzahl an parallel geöffneten Fenstern vermeidet diese Abhängigkeit und bietet einen grösseren Gestaltungsspielraum. Die Jugendlichen können entscheiden, wo sie sich wie stark engagieren und durch die Rücknahme von Aufmerksamkeit in einem Dialog bzw. durch die Herbeiführung von Kommunikationsabbrüchen wird vermieden, dass unangenehmer Stress auftritt. Der kurzfristige Nutzen des Chats kann durch dieses Verhalten reguliert werden.

Sonja: «Ich kann schnell schreiben. Wenn es zuviel werden, tu ich paar weg, aber drei, vier habe ich meistens schon».

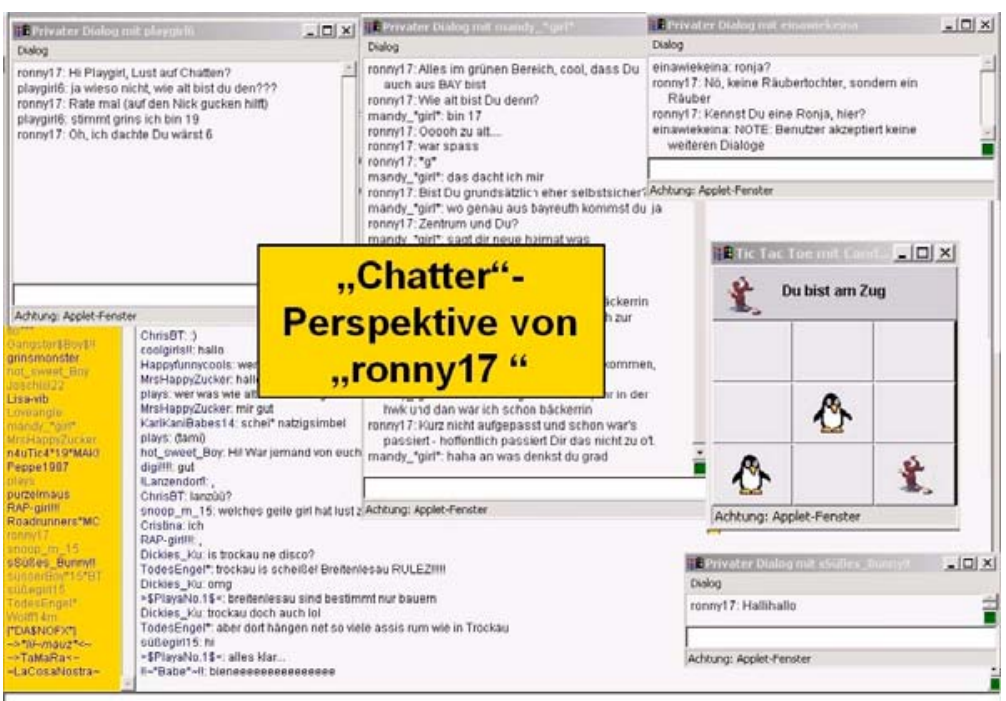

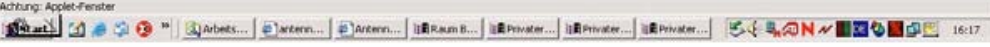
Abb. 2: Bildschirmansicht des Chatpartners »ronny17» mit vier geöffneten Dialogen und einem Spiel

Da insbesondere bei Erstkontakten die Verbindlichkeit eines Gesprächs als sehr gering eingestuft wird und da nicht mit Konsequenzen gerechnet werden muss, wenn der Kontakt abrupt abgebrochen wird, ist dieses Ver- 
halten im Chat häufig aufzufinden. Ursachen hierfür können äussere Faktoren (z. B. Abbruch der Internetnutzung nach Intervention von Eltern), Folge der Parallelkommunikation, aber auch das Desinteresse an einer Person bzw. Antipathie sein. Da es sich dem Einblick der Jugendlichen entzieht, warum ihnen die Aufmerksamkeit des Gegenübers nicht mehr zuteil wird, sind sie auf eigene Interpretationen angewiesen. Diese unklare Situation bietet auch aus der Perspektive des Einzelnen unterschiedliche Interpretationsmöglichkeiten:

Berücksichtigt man das Geschehen in den Dialogen, wird deutlich, dass das im Hauptfenster zu beobachtende Geschehen im Chat-Raum kaum dafür geeignet ist, die Kommunikation der Chat-Besucher untereinander zu erfassen. Die Handlungsmöglichkeiten des Chats eröffnen ausserhalb und in Kombination mit dem Hauptfenster individuelle Kommunikationspraktiken, die inhaltlich, hinsichtlich der Dauer und der Geschwindigkeit der Kommunikationssequenzen sowie im Hinblick auf die Zusammensetzung und die Anzahl der Chat-Partner variiert werden können.

Würde zur Untersuchung der Chatnutzung der Jugendlichen ausschliesslich die öffentliche Kommunikation im Hauptfenster berücksichtigt werden, die für alle Chatter lesbar ist, wäre ein Textkorpus gewählt, der in der Regel nicht der Perspektive des jugendlichen Chatbesuchers entspricht. Der aus der Perspektive des Lurkers bzw. des Beobachters beobachtbare Text und die so beobachtete Häufigkeit oder Geschwindigkeit, mit der sich ein Chatter in einem Chat-Hauptfenster engagiert, ist also nicht gleichzusetzen mit dessen tatsächlichem Engagement.

Nur wenn von den Chatbesuchern gegenseitig hinreichend viel Aufmerksamkeit aufgebracht wird und der Dialog für beide hinreichend viele Gratifikationen enthält, die eine Weiterführung des Gesprächs (in Konkurrenz mit gegebenen Alternativen) attraktiv erscheinen lässt, kommt ein längerer Austausch zu Stande. Für die Beteiligten kann sich daraus das Interesse entwickeln, über diesen ersten Kontakt hinaus wieder mit ihrem Gesprächspartner kommunizieren zu wollen. Die Jugendlichen im Chat haben diesbezüglich unterschiedliche Haltungen und Strategien, wie sie sich hinsichtlich dieser Fragestellung verhalten.

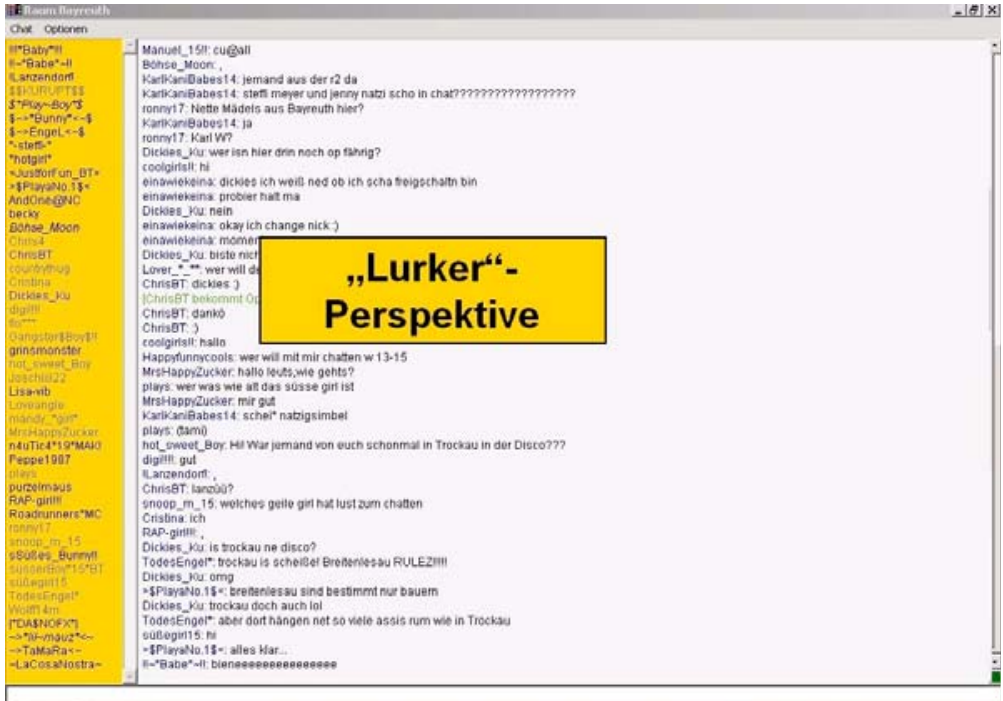

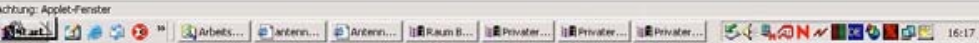

Abb. 3.: Zeitgleiche Bildschirmansicht der unbeteiligten Beobachters

Zwar ist ein Grossteil der befragten Besucher im Antenne-Bayern-Chat daran interessiert, andere Jugendliche kennen zu lernen, aber dies trifft nicht auf alle Besucher zu und die «Aufgeschlosseneren» haben unterschiedliche Vorstellungen über die Geschwindigkeit und die Modalität, mit denen dieser Kontakt verfestigt werden könnte.

Jugendliche, die ein Interesse haben, andere Chatter näher kennen zu lernen, signalisieren dies, indem sie sich mit dem Gesprächspartner wieder im Chat verabreden, E-Mail-Adressen austauschen und sich Fotos schicken, Handynummern austauschen, Homepage-Adressen weitergeben oder ein Treffen vereinbaren. Die individuelle Bereitschaft des Einzelnen für diese Handlungen ist ebenso wie die Deutung der Haltung des Gegenübers hierzu recht heterogen. Dies ist zum einen auf die unterschiedlichen subjektiven «Validierungstechniken» zurückzuführen, mit

${ }^{7}$ Da die jugendlichen Besucher des Antenne Bayern Chat 2003 auf das kostenpflichtige Angebot, ihren Nickname zu registrieren, verzichteten und der Chat-Raum stark frequentiert bzw. überfüllt war, war es 2003 schwieriger, eine Verabredung im Chat zu realisieren. 
denen die Angaben des Gegenübers vor dem Hintergrund der subjektiven Annahmen über Authentizität «generalized others» im Chat überprüft werden, und zum anderen auf die individuellen Ressourcen und Dispositionen für eine Kontaktaufnahme mittels anderer medialer Kanäle.

Im öffentlichen Web-Chat gelten andere Präsuppositionen und Maximen als in Alltagskonversationen. Höflichkeit und Zuverlässigkeit aber auch Wahrhaftigkeit, Richtigkeit und die Orientierung auf Verständigung können nicht als allgemein geteilte Maximen der am Chat beteiligten Personen unterstellt werden. Durch die Regelwerke und die Sanktionierungspraxen der Operatoren können die Prinzipien unter Bedingungen der internetbasierten Kommunikation nicht durch externen Druck etabliert werden und der Einzelne hat in dieser (auch spieltheoretisch interessanten) Konstellation nicht die Möglichkeiten und das Interesse, diese Kommunikationspraxis anders zu gestalten. So ist es auch nicht überraschend, wenn bei einigen Beteiligten und deren Beobachtern die Geltung anderer Handlungsmaximen angenommen wird.

Verena (14): «Ich glaub' zum Chatten gehört das ein wenig dazu, dass man ein wenig flunkert, in Anführungszeichen mal. Also ich sag' auch oft, dass ich 15 bin und nicht 14 oder so. Das find' ich gehört schon dazu.»

Wenn aber die ersten Kontakte im Chat intensiviert und in festere Beziehungen übergeführt werden sollen, werden (bereits im Chat) Kriterien angewandt, die denen der Alltagskommunikation entsprechen. Da unter den Bedingungen computervermittelter Kommunikation nur aufgrund der beobachteten Ereignisse in den Anzeigensystemen auf das medienbasierte Verhalten des Gegenübers und von diesem Verhalten auf Persönlichkeitsmerkmale geschlossen werden kann, die den Beteiligten für die Weiterführung von Kommunikation wichtig sind, ist eine Einschätzung sehr schwierig. Ein zentrales Kriterium, das für die Jugendlichen bedeutsam wird, ist die Einschätzung der Authentizität des Gegenübers. Da die Möglichkeiten der Maskierung und der Täuschung bekannt sind, betrachten einige Jugendliche die Selbstdarstellungen ihrer Gesprächspartner skeptisch:

Tanja: «Man beschreibt sich ja doch besser als man ist. »

Lena: «Ich glaube nicht, dass meine Chat-Partner ehrlich sind zu mir. Manche sagen, dass sie voll hübsch sind und dann sind die doch nicht so hübsch. »
Christian: «Ich glaube nicht, dass die Chat-Partner ehrlich sind. Die meisten schwindeln, aber ich nicht. »

Sonja: «Wenn ich jemand frage, wie er ausschaut, dann kommen immer dieselben Antworten. Immer! Es kann ja nicht jeder super ausschauen. »

Bereits eine Beschreibung, die zu sehr einem Klischee entspricht, kann dazu führen, dass die Glaubwürdigkeit der Aussagen in Frage gestellt wird und der Kontakt deshalb nicht weiter verfolgt wird. Um das Äussere der Chat-Partner vor einem Treffen zu überprüfen, fordern einige Jugendliche Fotos von ihren Gesprächspartnern an, die über Homepage, E-Mail, Handy übermittelt werden können. Da nicht jeder Jugendliche im Untersuchungszeitraum über eine entsprechende Ausstattung (Scanner, digitale Fotokamera, Fotohandy o. ä.) zur Erstellung einer Bilddatei verfügte, konnten einige solchen Forderungen nicht nachkommen, was von anderen wiederum als Beleg für Täuschung interpretiert wurde.

Der teilweise unklare bzw. unübersichtliche Adressatenbezug der Beiträge im Hauptfenster, die unterschiedliche Handhabung von Dialogen und Validierungstechniken erzeugen Kommunikationsabbrüche und begünstigen negative Erlebnisse. In ihrer Rolle als Chat-Partner sind die jugendlichen Internetnutzer (zumindest zu Beginn eines Kontakts) sehr austauschbar. Lange bleiben sie über die Integrität des Gegenübers im Unklaren und die Anonymität und die relative Sanktionsfreiheit im Chat begünstigen bei Einzelnen Diskrepanzen zwischen Aussendarstellung und Selbstwahrnehmung.

Die Jugendlichen müssen sich gegenseitig in mehreren Punkten Ehrlichkeit unterstellen, um sich näher kennen zu lernen. Damit bringen sie bereits im Chat aber ein »strengeres» Kriterium ein als im Regelwerk zu finden ist und dessen Einhaltung in diesem Kontext keine Verbindlichkeit beanspruchen kann. Die Motivation, wirklich andere Jugendliche kennen zu lernen, wird mit anderen, kurzfristig regulierbaren Bedürfnissen (angenehme Geschwindigkeit, Kurzweil, Spass etc.) vermischt und erfordert die Anpassung des eigenen Handelns an die Prinzipien des Chats.

Um einen angenehmen und unterhaltsamen Chat-Besuch zu haben, ist es wünschenswert, einen den individuellen Vorstellungen entsprechend temporeichen und kontinuierlichen Kommunikationsaustausch mit unterhaltsamen Chat-Partnern zu haben. Um dies zu gewährleisten, ist es nahe liegend, sich attraktiv darzustellen und mehrere Kommunikationspartner gleichzeitig bei der Stange zu halten. Ein Kontakt könnte jederzeit ab- 
brechen und da ist es praktisch, wenn noch andere Dialoge geöffnet sind. Diese Massnahmen, die einen unterhaltsamen Chat-Besuch wahrscheinlicher machen, begünstigen aber andererseits eine mangelnde Authentizität der Chat-Besucher und Unklarheit über die subjektive Relevanz der Gesprächssituation für das Gegenüber. Widmet er seine Aufmerksamkeit mir allein, oder umschwärmt er gleichzeitig jemand anderen? Diese Konstellation erschwert eine Vertiefung des Kontaktes und führt dazu, dass es eher unwahrscheinlich wird, dass längerfristige Kontakte entstehen.

\section{Medienkompetenz als Reflexion der Zielorientierung \\ eigenen Medienhandelns}

Dieser Zielkonflikt ist den Jugendlichen meist nicht klar, da über die eigene Internetnutzung eher wenig reflektiert wird. Die Ausbildung eines reflexiven Verhältnisses zur eigenen Mediennutzung ist - im Gegensatz zur Aneignung instrumenteller Fertigkeiten - offensichtlich keine notwendige Folge von Mediensozialisation und deshalb m. E. als besondere medienpädagogische Aufgabenstellung zu berücksichtigen.

Die vorliegenden Befunde deuten darauf hin, dass die 14- bis 16-jährigen Jugendlichen der Stichprobe die Chatbesuche und deren Folgen im Hinblick auf eigene Erwartungen daran wenig reflektieren, bewerten und ggf. korrigieren. Die Fähigkeit hierzu - reflexive Medienkritik als elementarer Bestandteil von Medienkompetenz - ist möglicherweise in diesem Alter ${ }^{8}$ nicht so stark ausgeprägt. Ein Ansatz für medienpädagogisches Handeln könnte darin bestehen, Reflexionsanlässe zu bieten, die Rationalität des Medienhandelns im Chat zu hinterfragen. Sollen aus den Chatbesuch wirklich neue Beziehungen resultieren, müssten die Chatbesucher ein höheres Mass an Höflichkeit und Zuverlässigkeit aber auch Wahrhaftigkeit, Richtigkeit und die Orientierung auf Verständigung einbringen, als dies von der Situation erfordert wird. Gerade bei Jugendlichen bedeutet dies die Selbstverpflichtung auf Handlungsmaximen, die in dieser Situation nicht durch unmittelbare äussere Sanktionen gestützt werden. Die Ausbildung einer normativen und selbstverantwortlichen Selbststeuerung ist bei den 14- bis 16-jährigen Internetnutzern aber noch nicht abgeschlossen. Indem medienpädagogische Angebote Anlässe bieten, sich hier verbindlich zu

\footnotetext{
${ }^{8}$ Ob es sich hier um ein altersspezifisches Phänomen handelt, müsste noch überprüft werden.

verorten, können sie die Identitätsentwicklung der Jugendlichen unterstützen.

\section{Literatur}

Baacke, Dieter: Medienkompetenz als Netzwerk. In: medien praktisch, Jg. 20. 2 (1996): S. 4-10.

Bahl, Anke. Zwischen On- und Offline. München: Kopaed Verlag, 1997.

Bruckman, Amy. Finding One's Own in Cyberspace. In: Technology Review I. (1996): S. 48-54.

Fend, Helmut. Die Entdeckung des Selbst und die Verarbeitung der Pubertät. Bern: Huber, 1994.

Fix, Tina. Generation @ im Chat. Hintergrund und explorative Motivstudie zur jugendlichen Netzkommunikation. München: Kopaed Verlag, 2001.

Hoffmann, Dagmar; Münch, Thomas. Mediale Aneignungsprozesse im Netz. In: medien + erziehung. Jg. 47, 5 (2003): S. 39-51.

Kammerl, Rudolf. Internetbasierte Kommunikation und Identitätskonstruktion. Hamburg. Verlag Dr. Kovac, 2005.

Kübler, Hans-Dieter. Wie anthropologisch ist mediale Kommunikation? In: medien praktisch, Jg. 25, 4 (2001): S. 11-20.

MpFS Medienpädagogischer Forschungsverband Südwest. JIM-Studie 2003. Jugend, Information, (Multi)Media. Baden-Baden: 2004.

Orthmann, Claudia. Kinder und Jugendliche auf der Suche nach Gesprächspartnern: Wer will chatten?. Chat-Kommunikation. Hrsg. v. Michael Beisswenger. Stuttgart: ibidem-Verlag, 2001: S. 279-304.

Orthmann, Claudia: Strukturen der Chatkommunikation. 24.3.2004 〈http://www.diss.fu-berlin.de/2004/78/index.html〉 (20.12.04).

Schatz, Tanja. Die individuelle Funktion des Chattens bei Jugendlichen. In: medien + erziehung. Jg. 47.5 (2003): S. 76-86.

Scherer, Helmut; Wirth, Werner. Ich chatte - wer bin ich? In: Medien \& Kommunikationswissenschaft, Jg. 50.3 (2002): S. 337-358.

Turkle, Sherry. Life on the screen. New York: Simon \& Schuster, 1995.

Wolf, Susanne; Bilandzic, Helena. (2003): Chatten als Kommunikationsspiel. In: Medien \& Kommunikationswissenschaft, 51. Jg. 4 (2003): S. 533-550. 